\title{
Illusions of apparent visual explosion and fusion
}

\author{
RONALD A. FINKE \\ State University of New York at Stony Brook, Stony Brook, New York
}

\begin{abstract}
Presentation of a display consisting of a whole, solid disk in alternation with a display in which sectors of the disk are radially and symmetrically displaced about its center creates the illusion that the disk is visually exploded into its parts, together with the illusion that the parts come together and are then fused. The minimum stimulus-onset asynchrony between the displays needed to produce these illusions is longer for apparent fusion than for apparent explosion, and in each case increases with increasing number of parts in the broken-up figures. Control experiments show that the fusion-explosion asymmetry cannot be attributed to differences in the direction of induced apparent motion (inward vs. outward), or to differences in the compactness of the displays.
\end{abstract}

Illusions of visual apparent movement are typically created by presenting, in rapid alternation, displays depicting the same object in adjacent locations or orientations (e.g., Kolers, 1972; Shepard \& Judd, 1976; Ullman, 1979; Wertheimer, 1912). At appropriate alternation rates, an observer has the sensation that the object is moving back and forth between the two positions or orientations. These illusions, which have been widely studied, are of particular interest to researchers in perception and cognition because they often help to reveal how the visual system interprets information about movement and other dynamic features of a visual stimulus (Attneave $\&$ Block, 1973; Farrell \& Shepard, 1981; Shepard \& Zare, 1983).

The present study reports an unusual kind of illusory motion, created by presenting a solid disk alternating with a display in which equal-area sectors of the disk are radially displaced away from its center. When the stimulusonset asynchrony (SOA) between the displays is increased sufficiently, an apparent explosion of the disk into its parts is seen, followed by an apparent inward motion of the parts which culminates in their apparent fusion, reforming the original disk. Unlike more typical forms of apparent movement, these two transitions are not symmetrical, in that a longer SOA is needed to produce smooth apparent fusion than to produce smooth apparent explosion. Some of the basic characteristics of these illusions are explored in the following experiments.

\section{EXPERIMENT 1}

\section{Method}

Subjects. Three observers, including the author, who were experienced in judging apparent motion served as volunteer subjects.

This research was supported by NIH Biomedical Research Grant 431H219L, awarded through the Research Foundation of the State University of New York at Stony Brook. I thank Jennifer Freyd, Evanne Casson, and Roger Shepard for helpful comments and suggestions, and Paul Choate for assistance in conducting the study. Requests for reprints should be addressed to the author at the Department of Psychology, State University of New York at Stony Brook, Stony Brook, New York 11794.
Procedure. Each observer was tested in five 1-h sessions, conducted on separate days over a two-week period. The first session counted as practice.

The stimulus pairs used in each session are shown in Figure 1. They were presented in alternation using a Gerbrands four-field tachistoscope at a viewing distance of $75 \mathrm{~cm}$ and at full illumination. For each of the stimulus pairs, the solid disk was $2.54 \mathrm{~cm}$ in diameter and was presented at the center of the viewing field. The two-, three-, and four-part patterns consisted of solid, equal-area sectors of the disk displaced $2.54 \mathrm{~cm}$ from the center of the field, symmetrically arranged as shown. In each configuration the sectors subtended the same visual angle of 5.82 deg.

The procedure for measuring the minimum SOA needed for producing each type of illusory motion was similar to that used by Shepard and Zare (1983): The displays were presented in alternation initially for durations of $150 \mathrm{msec}$ each, with no interstimulus interval. For all observers, this created an SOA sufficiently short that neither apparent explosion nor apparent fusion was smooth or continuous. At the beginning of each trial the experimenter indicated whether apparent explosion or apparent fusion was to be judged. The stimulus durations of the two displays were then increased in 10-msec increments until the observer reported that the illusory transformation did appear to be smooth and continuous. The SOA was then recorded, and the procedure was repeated on each subsequent trial. There were 30 trials in each session, yielding a total of 120 recorded observations per subject, with equal numbers of trials on which judgments of apparent fusion and apparent explosion were obtained, and equal numbers of trials on which the "ex-
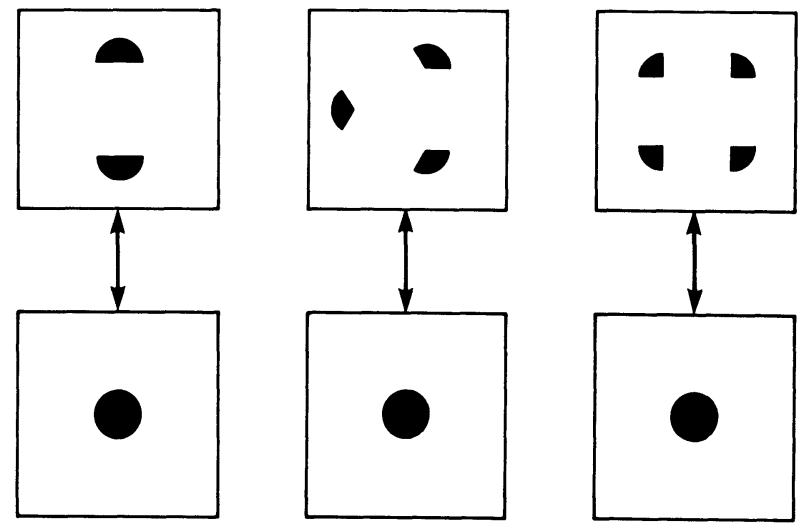

Figure 1. Stimulus pairs used to create illusions of apparent visual explosion and fusion in Experiment 1. 
ploded" pattern consisted of two, three, or four parts. The trials were conducted in random order, with a brief rest period in between to minimize fatigue. The data were analyzed both individually and across observers.

\section{Results}

Figure 2 presents the average minimum SOAs for each observer and condition, together with the group means. All three observers reliably needed a longer SOA in order to observe smooth apparent fusion than to observe smooth apparent explosion $(\mathrm{p}<.001$ for each observer), and longer SOAs as the number of parts increased $(\mathrm{p}<.001$ for each observer). A repeated-measures analysis of variance for the mean judgments across observers also showed that the minimum SOA needed for apparent fusion was significantly longer than that needed for apparent explosion $[F(1,2)=28.66, p<.05]$, and that both increased with increasing number of parts $[\mathrm{F}(2$, $4)=372.45, p<.001]$. In addition, the fusion-explosion difference increased with increasing number of parts, as revealed by a significant interaction $[\mathrm{F}(2,4=9.82, \mathrm{p}$ $<.05]$. A trend analysis on the effect of increasing the number of parts showed that only the linear trend was significant $[\mathrm{F}(1,4)=734.94, \mathrm{p}<.001]$. The standard errors of the individual means ranged from 3.13 to $8.05 \mathrm{msec}$.

\section{Discussion}

The results of this first experiment demonstrate that apparent visual explosion can be observed at a faster alternation rate than apparent visual fusion, and that slower alternation rates are required in each case as the number of parts increases. In addition, increasing the number of parts increases the fusion-explosion asymmetry.

It is tempting to interpret these results as reflecting some intrinsic difference in the way the visual system processes information about these two types of events. However, the asymmetry between apparent explosion and apparent fusion could also have been due to possible differences in how easily outward, as opposed to inward, apparent movement can be seen, or to differences in the relative compactness of the stimulus

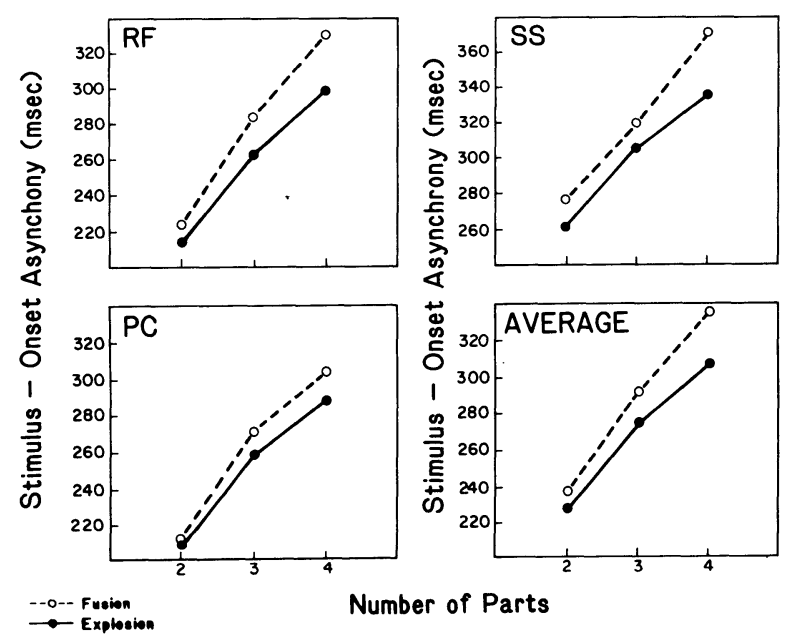

Figure 2. Minimum stimulus-onset asynchrony (SOA) needed to produce smooth apparent explosion and fusion as the number of parts increased (Experiment 1).
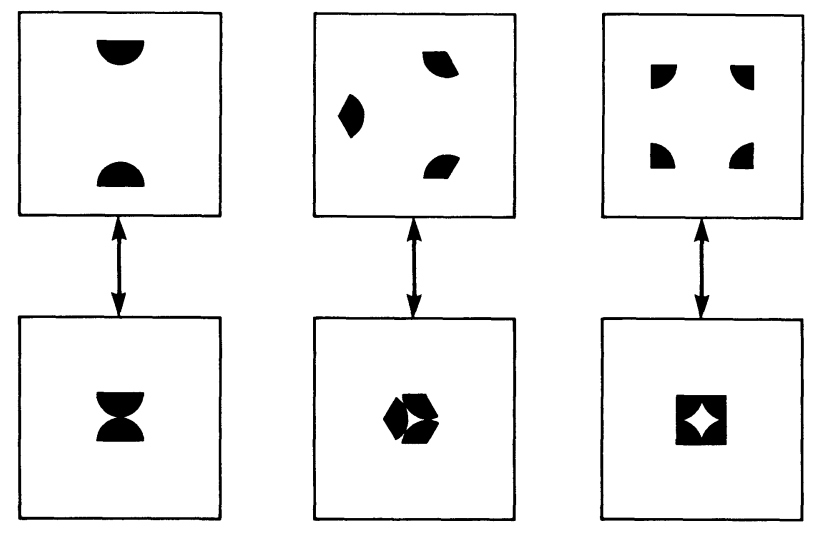

Figure 3. Stimulus pairs used to create illusions of apparent inward and outward motion of corresponding parts of patterns in Experiment 2.

displays. These alternative accounts were considered in the next two experiments, in which the same paths of apparent motion for the disk sectors were created, but without the appearance of fusion.

\section{EXPERIMENT 2}

\section{Method}

Subjects. Two of the observers from Experiment 1, plus a third trained observer (R.M.), who was unaware of the previous results, served as volunteer subjects.

Procedure. As shown in Figure 3, the stimulus pairs were modified by reversing each of the sectors, both in the original whole disk and in the patterns in which the parts had been displaced, so that the individual sectors would now appear to travel along the same paths as in Experiment 1, but would not appear to fuse. As before, the displaced parts subtended $5.82 \mathrm{deg}$ of visual angle. The observers were instructed, as the observers in Experiment 1 had been, to judge when the apparent inward and outward motions of the sectors were smooth and continuous. Except for the modification of the stimulus pairs, the procedure for Experiment 2 was identical to that for Experiment 1.

\section{Results and Discussion}

As in Experiment 1, increasing the number of parts resulted in a significant increase in the minimum SOA needed for producing smooth apparent motion, both within observers ( $p<.001$ for all three observers) and for the mean judgments across observers $[\mathrm{F}(2,4)=8.63$, $\mathrm{p}<$ .05]. However, in contrast to the results of Experiment 1, there was no effect of the direction of apparent motion ( $p>.10$ for all tests of significance), nor was there an interaction between the direction of motion and the number of parts $(F<1)$. These results, which were consistent for all three observers, are presented in Figure 4.

Trend analyses showed that the effect on the minimum SOA of increasing the number of parts was highly linear, as before $[F(1,4)=16.90, p<.05]$. However, the slopes of these functions were only about half as large as those in Experiment 1. The standard errors of the means were also smaller, ranging from 1.50 to $4.81 \mathrm{msec}$.

Each display in Experiment 2 was constructed such that the peripheral extent of the displaced sectors would be identical to that in the corresponding display in Experiment 1 . As a result, the distance between corresponding 
parts in the displays decreased slightly as the number of parts increased, due to small differences in how closely sectors in the compact displays could be arranged. (These differences are apparent in Figure 3.) While such differences could not account for the absence of an effect of the direction of apparent motion, they do present a possible confounding with the effects of increasing the number of parts. A third experiment was therefore conducted, in which the procedure of Experiment 2 was repeated but with the separation distance between corresponding parts held constant for all three patterns.

\section{EXPERIMENT 3}

\section{Method}

Subjects. The two observers who participated in both Experiments 1 and 2 also participated in this experiment. The third trained observer (K.B.) had not participated in either of the previous experiments, and was unaware of the results of those experiments.

Procedure. The procedure of Experiment 2 was followed exactly, except that the parts of each pattern in the expanded displays were displaced $2.54 \mathrm{~cm}$ from their corresponding parts in the compact displays. This was the displacement distance that had been used in creating the expanded displays in Experiment 1.

\section{Results and Discussion}

The results of this experiment, shown in Figure 5, were essentially identical to those of Experiment 2. Again, there was a significant effect on the minimum SOA of increasing the number of parts, both within observers $(p<.001$ for all three observers) and across observers $[\mathrm{F}(2,4)=$ $8.61, p<.05]$; but there was no effect of the direction of apparent motion ( $\mathrm{F}<1$ for all analyses), nor was there an interaction between direction of apparent motion and the number of parts $(F<1)$.

Also as in Experiment 2, the linear trend for the effect of increasing the number of parts was highly significant $[F(1,4)=17.23, p<.05]$, with minimum SOA increas-

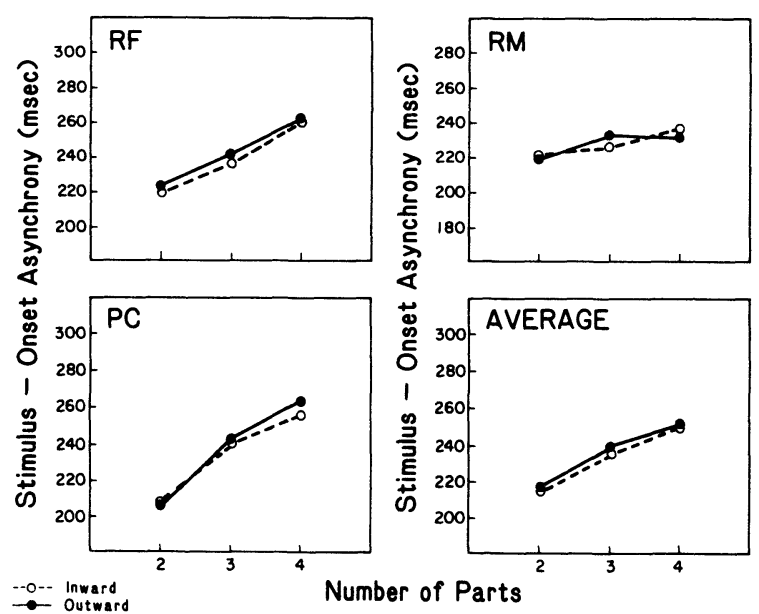

Figure 4. Minimum stimulus-onset asynchrony (SOA) needed to produce smooth apparent inward and outward motion of corresponding parts of patterns as the number of parts increased (Experiment 2).

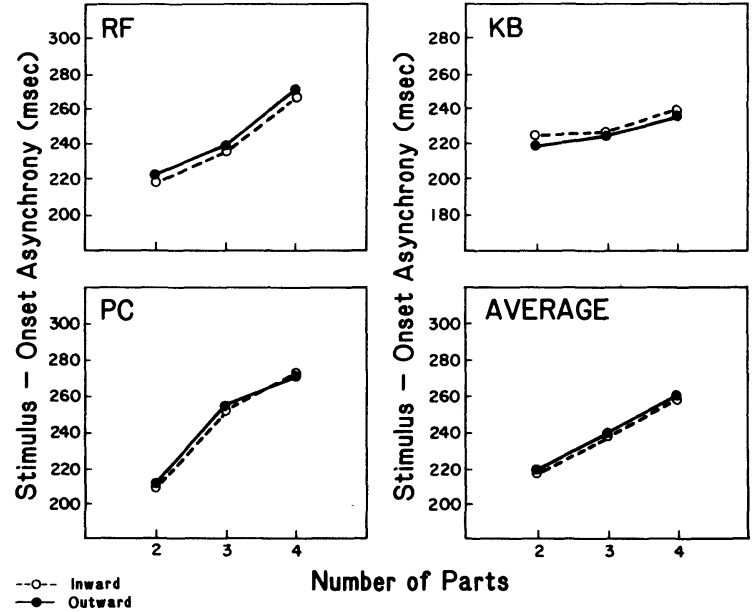

Figure 5. Minimum stimulus-onset asynchrony (SOA) needed to produce smooth apparent inward and outward motion of corresponding parts of patterns as the number of parts increased (Experiment 3).

ing at about half the rate of increase found in Experiment 1 . The standard errors, too, were similar to those in the previous experiment, ranging from 1.96 to 3.89 msec.

The combined results of Experiments 2 and 3, therefore, permit one to rule out direction of apparent motion (inward vs. outward) and differences in the relative compactness of the stimulus displays as explanations for the explosion-fusion differences in Experiment 1 . They also show that apparent radial motion of the individual sectors could only partially account for the effect of increasing the number of parts on the minimum SOA needed to produce apparent fusion and explosion.

\section{GENERAL DISCUSSION}

These exploratory studies reveal several significant characteristics of these illusions. The most striking characteristic is that the minimum SOA needed to produce the illusions is greater for apparent fusion than for apparent explosion, and that this difference increases as the number of parts into which the pattern is visually exploded increases. This asymmetry is evidently not due to the particular directions of apparent motion created, or to differences in compactness between the intact and visually exploded patterns.

These differences in apparent fusion and apparent explosion may be related to another type of asymmetry in apparent motion, recently reported by Freyd (1983). Using as inducing stimuli pairs of photographs depicting real-world action sequences, she found that subjects rated the quality of apparent movement in the forward direction as better than that in the backward direction across a wide range of SOAs. Freyd suggested that the visual system may be biased to favor natural, irreversible transformations, which are more likely to be perceived. If one regards the breaking up of an object into its parts as a more likely event than the spontaneous fusion of those parts, the present findings are at least consistent with those of Freyd.

It is useful to distinguish between these illusions and the phenomenon of "split motion," in which alternating presentations of single and multiple drawings of an object create the impression that duplications of the object move to several places at once, and then come back together (e.g., Wertheimer, 1912). While those illusory motions are commonly 
referred to as apparent "fission" and "fusion," respectively (Kolers, 1972), the objects are not truly seen to break up into their component parts, and the apparent motions are not asymmetrical in quality. Illusions involving split motion may therefore be fundamentally different from those reported here.

Another interesting feature of the present illusions is that the minimum SOA needed to produce them increases in proportion to the number of parts. This finding probably reflects the increased processing time needed as the inducing displays become more complex, although there are other possible explanations. For instance, increasing the number of parts may also increase the spatial frequency of the displays, which is known to influence the likelihood of seeing apparent motion (e.g., von Grünau, 1978). The effect of increasing the number of parts might also be explained in terms of the increased presence of subjective contours in the more complex patterns.

\section{REFERENCES}

Attneave, F., \& Block, G. (1973). Apparent movement in tridimensional space. Perception \& Psychophysics, 13, 301-307.

FARRELl, J. E., \& SHEPARD, R. N. (1981). Shape, orientation, and apparent rotational motion. Journal of Experimental Psychology: Human Perception \& Performance, 7, 477-486.
FreYD, J. J. (1983). The mental representation of movement when static stimuli are viewed. Perception \& Psychophysics, 33, 575-581.

Kolers, P. A. (1972). Aspects of motion perception. New York: Pergamon Press.

SHEPARD, R. N., \& JUDD, S. A. (1976). Perceptual illusion of rotation of three-dimensional objects. Science, 191; 952-954.

ShePard, R. N., \& ZARE, S. L. (1983). Path-guided apparent movement. Science, 220, 632-634.

Ullman, S. (1979). The interpretation of visual motion. Cambridge, Mass.: MIT Press.

VON GRÜNAU, M. W. (1978). Interaction between sustained and transient channels: Form inhibits motion in the human visual system. $\mathrm{Vi}$ sion Research, 18, 197-201.

Wertheimer, M. (1912). Experimentalle Studien über das Sehen von Bewegung. Zeitschrift fü Psychologie, 61, 161-265.

(Manuscript received for publication March 16, 1985.) 